\title{
Pleomorphic Adenoma of the Nasal Vestibule - A Rare Case
}

\author{
MA Matin ${ }^{1}$, Enamul Haque ${ }^{2}$, Md Asafuddoula ${ }^{3}$, Subroto Ghosh ${ }^{4}$, Mahmud Hossain ${ }^{5}$
}

\begin{abstract}
:
Pleomorphic adenomas arising in the nasal cavity are extremely uncommon, despite the large numbers of minor mucous and serous glands in the region. We present a rare case of a pleomorphic adenoma arising from the right nasal vestibule in a 65-year-old man complained of right nasal obstruction for 6 months. Anterior rhinoscopy showed a reddish, firm, polypoid mass in right nasal cavity which is free from the nasal septum and from the inferior tubinate but it was attached to the nasal vestibule. Flexible nasal endoscopy showed no extension of the mass towards the posterior choana or nasopharynx. The mass was completely excised from the nasal vestibule through intranasal approach and sent for histology which confirmed a pleomorphic adenoma with a predominant stromal component. The main treatment modality is surgical resection with histological clear margins. Recurrences and evolution to malignancy are not frequent, but long tern follow-up is recommended. In our case, the patient demonstrated satisfactory cosmetic results with no evidence of recurrence.
\end{abstract}

Keywords: Nasal vestibule. Pleomorphic adenoma, intranasal approach.

\section{Introduction}

Pleomorphic adenomas are the most common neoplasms of the salivary gland, occurring mainly in the parotid, submandibular and sublingual glands. These neoplasms affect females more than males and are commonly

1. Professor and Head of the Department of ENT\& HNS, Rajshahi Medical College

2. Asstt. Prof of ENT, Rajshahi Medical College

3. Professor of Pathology, Rajshahi Medical College

4. Asstt Professor ENT, Rajshahi Medical College

5. Asstt Registrar ENT, Rajshahi Medical College

Address for Correspondence: Professor M A Matin FRCS, Professor and Head of the Department of ENT \& HNS, Rajshahi Medical College, Bangladesh, Cell 01725897870, Email: matinfrcs@yahoo.com seen in the third to sixth decades of life. ${ }^{1}$ While pleomorphic adenomas are highly uncommon in the nasal cavity, approximately $80 \%$ of nasal pleomorphic adenomas are detected in the nasal septum, while the remaining $20 \%$ are found in the lateral wall or turbinate despite the fact that a significant proportion of mucous and serous glands is confined to the lateral nasal wall. ${ }^{2}$ The largest reported case series of intranasal pleomorphic adenomas are those of Spiros et al, with 40 cases. ${ }^{3}$ Compagno and Wong with 40 cases $^{4}$ and more recently, Suzuki et al with 41 cases. $^{5}$

The reason for the predilection of nasal pleomorphic adenomas to lateral nasal wall has been postulated by some authors. Stevenson suggested that remnants of the vomeronasal organ, an epithelium-lined duct in the cartilaginous nasal septum degenerated in early foetus, could be the reason for the 
appearance of these tumours in the nasal turbinate $^{6}$ Whereas, Ersner and Saltzman postulated that the precursors of the septal pleomorphic adenomas are ectopic embryonic epithelialised cells on the nasal septum mucosa, found during the migration of the nasal buds ${ }^{7}$ We present a rare case of a pleomorphic adenoma arising from the nasal vestibule.

\section{Case report:}

A 65 years old otherwise healthy man was presented to the Department of ENT and Head Neck Surgery with right sided nasal obstruction and occasional epistaxis for 6 months. He had no other otolaryngology symptoms. Anterior rhinoscopy showed a reddish firm, polypoid mass in right nasal cavity (Fig.-1).

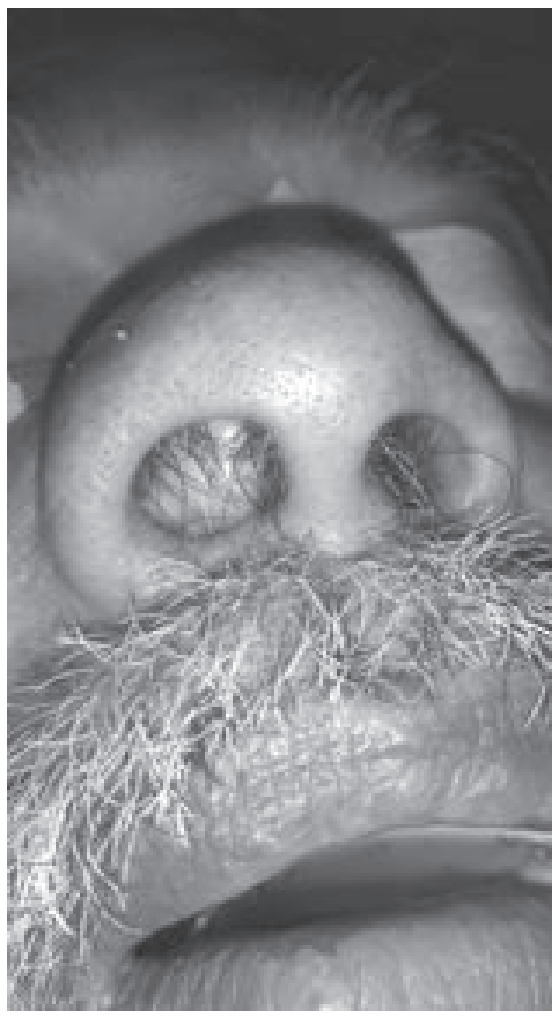

Fig.-1
The mass was free from the nasal septum and from the inferior turbinate but was attached to the right nasal vestibule with a brought base (Fig.-2).

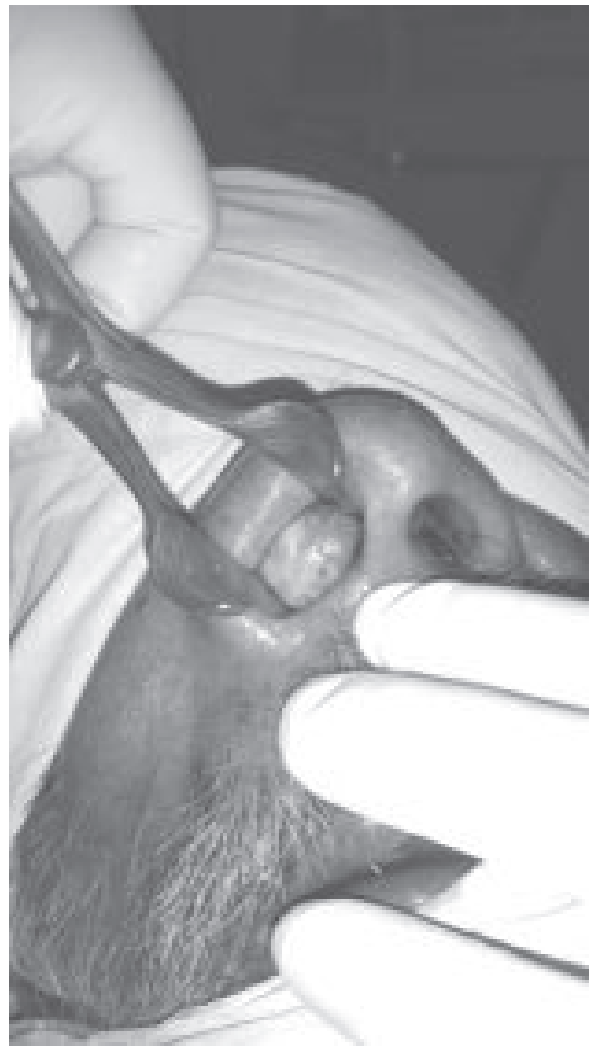

Fig.-2

Felixible nasolaryngoscopy showed no extension of the mass to the posterior choana or nasopharynx. There were no palpable cervical lymph nodes and no other abnormalities in the rest of the ear, nose and throat examination. As the mass was limited to the nasal cavity and attached laterally to the nasal vestibule so no further investigation like CT Scan of para-nasal sinuses was done apart from routine investigations for GA fitness. The whole mass was excised intranasally from the nasal vestibule using 
diathermy under general anaesthesia and sent for histopathology. The final histology showed a pleomorphic adenoma with epithelial and stromal component and appeared myxochondroid with no evidence of malignancy (Fig.-3).

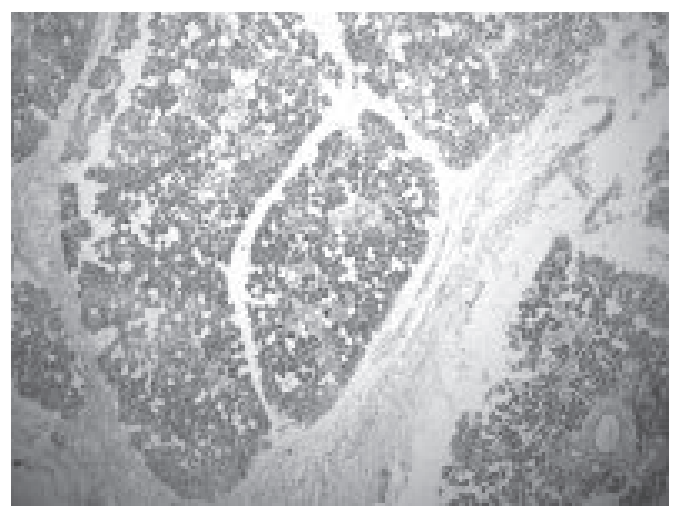

Fig.-2

The post-operative recovery was uneventful and he was discharged well.

\section{Discussion:}

Pleomorphic adenomas arising in the nasal cavity are extremely rare, despite the large numbers of minor mucous and serous glands in the region 4 Patients often seek medical attention because of nasal obstruction, epistaxis or the presence of a nasal mass.

Histologically, pleomorphic adenomas of the nasal cavity differ in certain characteristics. The nasal cavity tumours have greater cellularity and contain more epithelial components than the chondroid, myxoid and collagenous stromal components that are seen in pleomorphic adenomas of the parotid gland ${ }^{1}$. The relatively low rate of recurrence is attributed to diminished amount of myxoidstroma of intranasal tumours $(7.5 \%){ }^{4}$

Motoori et al. ${ }^{8}$ reported a case of recurrence in which the nasal pleomorphic adenoma had a cellular pattern with many areas of myxoid stromal predominance. It is postulated that the myxoidstroma could easily be spilled into the surgical field, providing a focus for recurrence.$^{4}$ Interestingly, the pleomorphic adenoma in this case has stromal predominance and resembles a typical adenoma of the parotid gland more closely than one of the nasal cavity.

The main treatment modality is complete surgical resection with histological clear margins. Recent literature has advocated the effectiveness of conservative excision via an intranasal endoscopic approach in eliminating disease with no recurrence on follow up. ${ }^{9,10}$ However, histologically the pleomorphic adenoma in these cases showed a predominance of epithelial rather than stromal elements.

The surgical approach should depend on the size, location and extension of the tumour. In this case, intranasal approach was chosen because the nasal tumour base was attached to the nasal vestibule without any extension to the sinuses or towards the nasopharynx.

It is a well known fact that there is always risk of recurrence, regardless of where the tumour arises from. The recurrence rate of pleomorphic adenoma depends almost on the sufficiency of the initial excision. Surgery for recurrent tumours often does not produce desirable results. Hence, good exposure is necessary to ensure complete excision of the tumour, more so when its cellular pattern shows stromal predominance. ${ }^{11}$

Given the rarity of the intranasal pleomorphic adenoma and heterogeneity of its behaviour, no standard treatment algorithm has been established. Nevertheless, post-operative follow up examination is mandatory in view of its potential for recurrence ${ }^{1}$ In our case, the patient demonstrated satisfactory cosmetic results with no evidence of recurrent disease during subsequent follow up visits. 


\section{Conclusion:}

In summary, pleomorphic adenomas are rare tumours of the nasal cavity. They have a higher cellular and lower stromal component compared to their major salivary gland counterparts and may be misdiagnosed at an early stage leading to more aggressive treatment. In view of the potential for tumourrecurrence,long term follow-up and careful examination of the nose with flexible or rigid endoscope are necessary

\section{References:}

1. Tan SH, Subranamium MM, Liu J, Chong KB, Loke D Pleomorphic adenoma of the lateral nasal wall: A case report. Egyptian Journal of Ear,Nose, Throat and Allied Sciences 2012;13(2):99-101

2. Batsakis J.Tumours of the head and neck: Williams and Wilkins, Baltimore. 1984:76-99

3. Spiro RH, Koss LG, HadidoSL.Tumours of minor salivary origin, a clinicopathological study of 492 cases. Cancer 1973; 31:117-129

4. Compango J, Wong RT. Intranasal mixed tumours (pleomorphic adenomas): aclinicopathologic study of
40 cases. Am J Clin Pathol.1977; 68: 213-218

5. Suzuki K, Moribe K, Baba S. A rare case of pleomorphic adenoma of lateral wall of nasal cavity, with special reference of statistical observation of pleomorphic adenoma of nasal cavity in Japan.Nippon J ibiinkoka Gakkai Kaiho.1990; 93:740745

6. Stevenson HN. Mixed tumour of the septum. AnnOtolRhinol Laryngol. 1932:41:563-570

7. Ersner MJ,Saltzman M.A mixed tumour of the nasal septum, report of a case.Laryngoscope 1944; 54:287-296

8. Motoori K, Takano H, Nakano K. Pleomorphic adenoma of the nasal septum: MRfeatures. AJNR AM J Neuroradiol.2000:21(10):1948-1950

9. Jackson LE, Rosenburg SI. Pleomorphic adenoma of the lateral nasal wall. Otolaryngol Head Neck Surg 2002;127:474-476

10. Sciandra D, Dispensza F, Porcasi, et al.Pleomorphic adenoma of the lateral nasal wall:casereport.Acta Otorhinolaryngolgica Itilica2008;28:150-153

11. MalletK J, Harrison MS. The recurrences of salivary gland tumours. J Laryngol Otol 1971:85:439-448 